\title{
Sesgos cognitivos y uso del e-gobierno. Una perspectiva conductual ${ }^{1}$
} Cognitive biases and use of e-government. A behavioral perspective

\section{NOTA BIOGRÁFICA}

\author{
Cayetano Medina-Molina \\ Universidad Isabel I \\ cayetano.medinamolina@ui1.es
}

Cayetano Medina-Molina es Licenciado en Ciencias Económicas y Empresariales (Universidad de SeviIla), Licenciado en Investigación y Técnicas de Mercado (Universidad de Sevilla), Doctor por la Universidad Nacional de Educación a Distancia. Profesor del Centro Universitario San Isidoro y Universidad Isabel I. Su investigación se centra en el marketing público y la adopción de servicios digitales.

\section{Manuel Rey-Moreno \\ Universidad de Sevilla mrmoreno@us.es}

\section{NOTA BIOGRÁFICA}

Manuel Rey-Moreno es Licenciado en Ciencias Económicas y Empresariales (Universidad de Sevilla) y doctor en Ciencias Económicas y Empresariales por la Universidad de Sevilla (Universidad de Sevilla). Catedrático de Escuela Universitaria (Administración de Empresas y Marketing). Director de la Cátedra Metropol Parasol (US). Su investigación se centra en las aplicaciones sectoriales del marketing y el marketing turístico.

\section{Benito Pérez-González Universidad Isabel I benito.perez@ui1.es} NOTA-BIOGRÁFICA

Benito Pérez-González es Licenciado en Ciencias Económicas y Empresariales y Doctor en Economía. Durante diez años desarrolla su carrera profesional en Ernst \& Young. Coordinador y profesor en la Universidad Isabel I. Su investigación se centra en las aplicaciones sectoriales del marketing y la economía del deporte.

Ramón Rufín

Universidad Nacional de Educación a Distancia rrufin@cee.uned.es

\section{NOTA-BIOGRÁFICA}

Ramón Rufín, Economista, es Profesor Doctor en la UNED, donde dirige el Grupo de Investigación de Marketing. Fue también Técnico Superior del Banco Exterior de España y Experto Asesor para la Administración General del Estado. Sus líneas de investigación actuales tienen que ver con el consumo cultural y turístico y con el marketing público.

1 El presente trabajo forma parte del proyecto de investigación Estrategia omnicanal en la distribución de servicios públicos financiado por el Instituto Nacional de Administración Pública. 


\begin{abstract}
RESUMEN
El e-gobierno se presenta como una herramienta al servicio de la mejora de la eficacia y eficiencia en la prestación de los servicios públicos. Pero, pese a los esfuerzos por lograr una adopción generalizada del e-gobierno, éste presenta unas tasas de uso inferiores a las anheladas. Si bien dicho fenómeno ha sido estudiado desde diferentes perspectivas, en el presente trabajo se presenta la perspectiva conductual como enfoque que responda a tal reto. Para ello, se analiza la relación entre diferentes sesgos cognitivos vinculados a la tendencia del statu quo (hábito, inercia y resistencia) con la intención (de uso para los usuarios y de continuación para los usuarios). Los datos fueron captados tras un trabajo cualitativo (dos sesiones de grupo) y un trabajo cuantitativo (923 cuestionarios), dirigido a dos muestras diferentes de ciudadanos (no-usuarios y usuarios del e-gobierno). Los resultados constatan la presencia de la tendencia al statu quo en el uso del e-gobierno. Mientras en el caso de los no-usuarios del e-gobierno se encuentran presentes los sesgos cognitivos analizados, llevando a dilatar su uso, entre los usuarios del e-gobierno carece de relevancia el hábito, por lo que se mantienen en su uso como canal de acceso a los servicios públicos.
\end{abstract}

\title{
PALABRAS CLAVE
}

Administración pública conductual; e-gobierno; hábito; inercia; resistencia.

\begin{abstract}
E-government is conceived as a tool to improve efficiency and effectiveness in the provision of public services. However, in spite of the efforts to achieve a widespread adoption of e-government, it has lower usage rates than expected. Although this fact has been studied from different viewpoints, in the present work the behavioral perspective is presented as an approach that may answer to this challenge. To get it, the relationship between different cognitive biases linked to the SQB (habit, inertia and resistance) with the intention (of use for users and continuation for users) is analyzed. The data were collected after a qualitative (two group sessions) and a quantitative work (923 questionnaires), aimed at two different samples of citizens (non-users and users of e-government). The results confirm the presence of the trend towards the status quo in the use of e-government. Besides, while in the case of non-users of e-government the cognitive biases analyzed are present, leading to delay its use, among the users of e-government habit lacks relevance, so they remain in use as a channel of access to public services.
\end{abstract}

\section{KEYWORDS}

Behavioural public administration, e-government, habit, inertia, resistance.

\section{SUMARIO}

1. INTRODUCCION. 2. LA RESISTENCIA Y LAADOPCIÓN DE SISTEMAS DE INFORMACIÓN. 2.1. MODELOS EXPLICATIVOS DE LA ADOPCIÓN DEL E-GOBIERNO. 2.2. LA ADMINISTRACIÓN PÚBLICA COMPORTAMENTAL, LA TENDENCIA AL STATU QUO. 2.3. LAS CAMPAÑAS PÚBLICAS DE CAMBIO DE COMPORTAMIENTO. 3. MÉTODOS. 3.1. MODELO DE INVESTIGACIÓN E HIPÓTESIS. 3.2. MEDICIÓN DE LAS VARIABLES. 3.3. MUESTRA Y DATOS. 3.4. ANÁLISIS DEL MODELO DE MEDIDA. 3.5. LIMITACIONES. 4. RESULTADOS. 4.1. ANÁLISIS DEL MODELO ESTRUCTURAL. 4.2. ANÁLISIS DE LOS RESULTADOS. 5. DISCUSIÓN DE LOS RESULTADOS. 6. CONCLUSIONES, LIMITACIONES Y FUTURAS LÍNEAS DE INVESTIGACIÓN.

\section{INTRODUCCION}

La oferta de servicios públicos a través del e-gobierno se vincula al logro de una prestación optima apoyada en estrategias multicanal, que coordinan canales de prestación de servicio tratando de permitir a ciudadanos y organizaciones elegir su canal preferido para interactuar con la administración (van Veenstra y Janssen, 2010). En esta línea, los gobiernos se embarcan en el desarrollo de aplicaciones de e-gobierno, y aunque son numerosos los beneficios que se le identifican, muchos de ellos fallan en el logro de sus objetivos, sabiéndose aún poco acerca de los factores que determinan su éxito o fracaso (Luna-Reyes et al., 2009). Dicha estrategia se enfrenta a retos significativos, ya que en el caso de no tener presentes las nece- 
sidades de ciudadanos y organizaciones los canales ofrecidos podrían resultar insuficientemente empleados o completamente rechazados (Nygren et al., 2014).

En España se presenta una situación paradójica, ya que pese a que la satisfacción con el e-gobierno es superior a la correspondiente al canal presencial, éste último sigue siendo el preferido por los ciudadanos para realizar sus trámites o gestiones con las administraciones públicas. La administración presencial no sólo es la preferida, sino que además ha aumentado el volumen de ciudadanos que acceden a las oficinas públicas (80\%), seguido, a gran distancia, por la e-administración (48\%) (AEVAL, 2016).

TABLA 1. EVOLUCIÓN USO INTERNET INTERACCIÓN CON LAS ADMINISTRACIONES PÚBLICAS

\begin{tabular}{|c|c|c|c|c|c|c|}
\hline & 2012 & 2013 & 2014 & 2015 & 2016 & 2017 \\
\hline Porcentaje de españoles que han utilizado Internet trato con las AAPP & 45,0 & 44,0 & 49,0 & 49,4 & 50,1 & 52,4 \\
\hline $\begin{array}{l}\text { Porcentaje de españoles que han utilizado Internet envío formularios } \\
\text { cumplimentados }\end{array}$ & 22,3 & 23,5 & 28,7 & 30,0 & 32,3 & 33,2 \\
\hline
\end{tabular}

Fuente: ONTSI (2017). http://www.ontsi.red.es/ontsi/sites/ontsi/files/Indicadores\%20Destacados\%20\%28octubre\%202017\%29_0.pdf.

En el caso español, parece que nos enfrentamos a una resistencia al uso del e-gobierno por parte de determinados grupos de ciudadanos, resultando especialmente llamativo el caso de los usuarios indirectos ${ }^{2}$ y no usuarios del e-gobierno. Así, mientras los usuarios indirectos reducen la posibilidad ${ }^{3}$ de realizar algún trámite a través del e-gobierno, $41 \%$ en 2013 , 33\% en 2014 y $31 \%$ en 2015 , el porcentaje de no usuarios que ve bastante o muy probable que lo utilicen en un futuro no muy lejano son $9 \%$ en $2012,11 \%$ en 2013 , $13 \%$ en 2014 y $13 \%$ en 2015 (AEVAL, 2016), por lo que parece que aquellos que no hacen un uso directo del e-gobierno no se plantean hacerlo en un futuro próximo. No obstante, de acuerdo con los datos de la ONTSI (2017), en los últimos años aumenta de forma sostenida el porcentaje de españoles que emplean Internet para tratar con la Administración y para el envío de formularios completados, datos que se alinean a las tasas que plantea Eurostat.

TABLA 2. USO DEL E-GobiERNO EN ESPAÑA

\begin{tabular}{|c|c|c|c|c|c|c|c|c|c|}
\hline \multicolumn{10}{|c|}{ Porcentaje de individuos (usuarios) respecto del total (de la muestra) } \\
\hline & 2008 & 2009 & 2010 & 2011 & 2012 & 2013 & 2014 & 2015 & 2016 \\
\hline Interacción con autoridades & 31 & 34 & 38 & 38 & 44 & 44 & 49 & 49 & 50 \\
\hline Obtener información & 30 & 33 & 37 & 36 & 42 & 41 & 46 & 45 & 47 \\
\hline Descargar formularios & 19 & 20 & 24 & 24 & 28 & 29 & 34 & 34 & 37 \\
\hline Remitir formularios completos & 12 & 11 & 16 & 17 & 22 & 24 & 29 & 30 & 32 \\
\hline
\end{tabular}

Porcentaje de individuos que han usado Internet el último año

\begin{tabular}{|c|c|c|c|c|c|c|c|c|c|}
\hline Interacción con autoridades & 53 & 55 & 58 & 56 & 61 & 59 & 63 & 62 & 62 \\
\hline Obtener información & 52 & 53 & 56 & 53 & 58 & 56 & 60 & 57 & 58 \\
\hline Descargar formularios & 32 & 32 & 36 & 35 & 40 & 40 & 44 & 43 & 45 \\
\hline Remitir formularios completos & 20 & 18 & 25 & 25 & 31 & 32 & 37 & 38 & 38 \\
\hline
\end{tabular}

Fuente: Eurostat $h$ ttp://ec.europa.eu/eurostat/tgm/table.do?tab=table\&init=1\&language=en\&pcode=tin00013\&plugin=1.

Una premisa que subyace bajo los estudios que explican el comportamiento vinculado al uso de las TIC es que se trata de un comportamiento intencional basado en decisiones conscientes o planeadas del usuario. Pero podrían seguir un patrón de habitualidad basado en secuencias de comportamiento repetidas automáticamente bajo rasgos del entorno más que por premisas conscientes, reclamándose la atención a los mecanismos inconscientes en la investigación relativa a la toma de decisiones de los consumidores (Bhattacherjee y Lin, 2015).

2 Son usuarios indirectos aquellos que, si bien no han empleado internet por sí mismo en los últimos 12 meses, han solicitado a otra persona que busque en Internet algún tipo de información o que realice alguna consulta, trámite o gestión relacionado con la Administración Pública o algún centro público.

${ }_{3}$ Porcentaje que ve muy o bastante probable la posibilidad de realizar en un futuro no muy lejano algún trámite a través de la Administración Electrónica. 
Es decir, pese a que los ciudadanos reconocen los beneficios vinculados al e-gobierno, el canal presencial sigue siendo el preferido para acceder a los servicios públicos, por lo que nos encontramos con un gap entre las actitudes positivas de los usuarios hacia determinados canales de prestación de servicios y el uso de los mismos, el denominado gap «actitud-comportamiento» (Moraes et al., 2012). Por todo ello un primer paso para una adopción generalizada de e-gobierno sería identificar aquellos elementos que llevan a resistirse al mismo (Ebbers y van Dijk, 2007). Cualquier resistencia a un pensamiento «nuevo» planteado por el gobierno es un desafío que debe ser reconocido y abordado (OECD, 2015). Aquellos que pretendan conocer las preferencias y decisiones en el ámbito público deberían completar sus modelos con elementos que tengan presente los sesgos cognitivos, cuyo estudio se vincula la aplicación del enfoque conductual en el sector público, la denominada administración pública conductual (Grimmelikhuijsen et al., 2016; Moynihan y Lavertu, 2012).

La aplicación de los conocimientos conductuales en el diseño e implementación de intervenciones de mercado no son una herramienta o enfoque totalmente nuevo. Éstos han sido utilizados no sólo por parte del sector privado sino también por los gobiernos y más aún por los reguladores (OECD, 2015; 2017). Por ello, se indica un elevado potencial a la aplicación de la economía conductual en el cambio de comportamiento de los agentes en la arena pública, habiéndose recomendado para modificar las pautas de comportamiento y las elecciones de los ciudadanos (OECD, 2017).

La administración pública conductual toma prestado y extiende teorías procedentes del campo de la psicología, incluyendo el liderazgo público y la motivación, la transparencia, la competencia y elección de servicios públicos y la confianza de los servidores públicos (Grimmelikhuijsen et al., 2016; Jilke, Van de Walle y Kim, 2016; OECD, 2015, 2017). Los gobiernos están aplicando los principios conductuales derivados de las ciencias sociales y del comportamiento que incluyen la toma de decisiones, la psicología, la ciencia cognitiva, la neurociencia y el comportamiento organizacional y grupal, con el objetivo de hacer que las políticas públicas funcionen mejor (OECD, 2017).

El uso del conocimiento vinculado al enfoque conductual puede ayudar a que los ciudadanos tomen mejores decisiones, al tiempo que favorecer un diseño e implantación más efectivo de las políticas. Al hacer que el gobierno sea más efectivo y se centre en los ciudadanos, las percepciones conductuales pueden ayudar a recuperar la confianza de los ciudadanos en la capacidad de los gobiernos para resolver sus problemas de la ciudadanía (OECD, 2015).

El objetivo del presente trabajo es explicar, desde el enfoque de la administración pública conductual, las intenciones -de uso o continuación en el uso- vinculadas al uso del e-gobierno. Para ello, se estudiará el papel jugado por determinados sesgos cognitivos (hábito, inercia y resistencia) sobre la intención de comportamiento vinculada al e-gobierno. Dicho análisis se realizará en dos muestras, una de no-usuarios del e-gobierno y otra de usuarios, para poder comparar el dispar efecto que los citados sesgos cognitivos tienen sobre la intención de uso y de continuación, en cada caso.

\section{LA RESISTENCIA Y LA ADOPCIÓN DE SISTEMAS DE INFORMACIÓN}

\subsection{Modelos explicativos de la adopción del e-gobierno}

La consolidación de Internet supone un factor de cambio de lo que el gobierno hace y cómo lo hace, habiéndose identificado cinco áreas donde ayuda a conseguir una mejor administración (INAP, 2009: 21), entre las que dos se vinculan con la forma en la que los servicios públicos resultan accesibles a los ciudadanos (1) e-gobierno orientado al usuario, haciendo los servicios electrónicos más receptivos a las necesidades de los ciudadanos; (2) distribución multicanal, mejorando los vínculos entre servicios tradicionales y electrónicos. En el análisis del e-gobierno se puede optar por una perspectiva amplia, que supone tener presente los cinco elementos antes indicados, o bien, decantarnos por una visión en la que el foco se centre en la distribución de servicios públicos (Medina-Molina, Rey-Moreno y Pérez-González, 2017). Dichas ópticas favorecen el surgimiento de términos similares como e-administración y e-gobierno, que presentan matices diferenciadores. Una primera óptica indica que el e-gobierno personifica la visión de una lógica global de la administración que actúa como catalizadora de su transformación (INAP, 2009), próxima al término e-administración entendido como una visión del e-gobierno extendida a una reconfiguración de las estructuras de la administración y de los servicios públicos (Pont, 2016). Una segunda óptica del e-gobierno, más concreta, se basa en el principio de habilitar a los usuarios el acceso a la información y servicios de la administración, cuando 
y como ellos quieran mediante diversos canales, Internet inclusive (INAP, 2009). Es bajo esta segunda perspectiva bajo la que se va a aplicar el concepto e-gobierno en el presente trabajo.

El estudio del e-gobierno desde la perspectiva de la demanda aborda los factores que determinan su aceptación, partiéndose de los modelos clásicos de adopción bajo el supuesto de que aquellos factores que desempeñan un papel importante en los modelos del comportamiento del consumidor en línea afectarán también a su adopción. El modelo clásico explicativo del comportamiento del consumidor en Internet más ampliamente aceptado es el Technology Acceptance Model (TAM), que constituye una adaptación de la Theory of Reasoned Action (TRA) centrada en la conducta de uso de nuevas tecnologías. El TAM reemplaza las creencias definidas en la TRA por 2 elementos - utilidad y facilidad de uso percibida-, bajo el supuesto de que influyen en la formación de actitudes y, en consecuencia, en la intención y conducta de un individuo acerca del uso de la tecnología. Posteriormente el modelo de la Diffusion of Innovations (DOI) establece que el lanzamiento de una innovación al mercado se modela como un proceso de captación de información y reducción de incertidumbre con la intención de evaluar la tecnología, identificando 5 elementos que pueden influirla: ventaja relativa, complejidad, compatibilidad, posibilidad de prueba y facilidad de observación de sus beneficios. Venkatesh, Morris, Davis y Davis (2003) sintetizan los intentos por explicar la adopción de nuevas tecnologías con el desarrollo del modelo Unified Theory of Acceptance and Use of Technology (UTAUT), que plantea la existencia de 4 factores determinantes en la aceptación y uso de las nuevas tecnologías: expectativa de resultado; expectativa de esfuerzo; influencia social; y condiciones facilitadoras. El modelo UTAUT plantea que la expectativa de resultado, la expectativa de esfuerzo e influencia social afectan a la intención de uso, mientras que esta última y las condiciones facilitadoras determinan su uso efectivo (Medina, Rufin Moreno y Rey, 2013)

Recientemente, Dwivedi, Rana, Janssen, Lal, Williams y Clement (2017) desarrollan el Unified Model of Electronic Government Adoption (UMEGA). Dicho modelo responde a la necesidad de tener presente las particularidades asociadas a la incorporación de las TIC en el sector público, entre ellas, la compleja estructura de objetivos de la administración y la realización de múltiples funciones, requiriéndose tener presente elementos que representen perspectivas específicas del e-gobierno, como pueden ser la confianza, el riesgo, la seguridad, la transparencia y la privacidad. Asimismo, pese a que el sector público goce de autoridad para imponer el uso del e-Gobierno, su aceptación voluntaria conducirá a una utilización optimizada. UMEGA postula cuatro determinantes directos de la actitud (expectativa de resultado, expectativa de esfuerzo, influencia social y riesgo percibido), dos determinantes directos de la intención de comportamiento (condiciones y actitud facilitadoras) y un determinante directo de la expectativa de esfuerzo (condiciones facilitadoras). De acuerdo con este modelo, la actitud juega un papel mediador clave en la adopción de un sistema de e-gobierno.

Dos elementos confluyentes provocan la necesidad de desarrollos en los modelos explicativos de la intención de comportamiento de los usuarios de los servicios públicos digitales. En primer lugar, la incorporación de nuevos canales de distribución lleva a tener presente el juego entre las preferencias hacia los mismos, en lo que se denomina estrategia multicanal u omnicanal dependiendo del enfoque desde el que se diseñen (Medina, 2017). En segundo lugar, conocer las preferencias y decisiones en el ámbito público requiere completar los modelos explicativos de la adopción con elementos que tengan presente los sesgos cognitivos, vinculados a la aplicación del enfoque conductual en el sector público, la denominada administración pública conductual (Grimmelikhuijsen et al., 2016; Medina-Molina, Rey-Moreno y Pérez-González, 2017; Moynihan y Lavertu, 2012).

Se propone el enfoque de la administración pública conductual como el análisis de la administración pública desde la perspectiva micro de las actitudes y comportamientos individuales, partiendo de las bases psicológicas que subyacen bajo los mismos. Entre sus ámbitos de aplicación se incluye la prestación de servicios digitales ya que se recomienda su uso en el sector público para modificar las pautas de comportamiento y elecciones de los ciudadanos (Grimmelikhuijsen et al., 2016; OECD, 2017).

\subsection{La Administración Pública Comportamental, la tendencia al statu quo}

La economía parte de la creencia de que el comportamiento de los agentes puede explicarse asumiendo que éstos poseen unas preferencias estables y bien definidas, realizando elecciones racionales en base a las mismas. Estos modelos clásicos de la racionalidad suponen la base más empleada para comprender la toma de decisiones. La teoría económica neoclásica supone que cuando el consumidor se enfrenta a una situación de elección, se decantará por la nueva alternativa si alguna de las nuevas opciones excede la utilidad de la alternativa en posesión (Kahneman et al., 1991; Moynihan y Lavertu, 2012). 
Simon (1955) desarrolla el concepto de racionalidad limitada para explicar que la riqueza de información provoca pobreza de atención y dificulta la toma de decisiones. Los trabajos sobre la racionalidad limitada fueron fundamentales para la aparición de la economía conductual, también referida como economía comportamental. Pese a que algunas teorías reconocen los procesos cognitivos como los conductores del comportamiento, en la práctica numerosos comportamientos se producen con muy poca deliberación cognitiva (Moraes et al., 2012). La creciente abundancia de información y de opciones favorece este proceso de toma de decisiones intuitivo y automático. Por todo ello los ataques al modelo del actor racional vienen de la propia economía, desde el enfoque de la economía conductual (Grimmelikhuijsen et al., 2016). Partiendo de la psicología, señala que las personas poseen una racionalidad imperfecta y que descansan en reglas heurísticas para tomar las decisiones. Los economistas conductuales reconocen la fragilidad humana -su irracionalidad y sus errores sistemáticos- y aprovechan los avances de la psicología en beneficio de la toma de decisiones (Ariely, 2008), proporcionando estrategias de actuación que favorezcan una toma de decisiones más favorables. Los economistas conductuales parten de las limitaciones de la economía neoclásica resaltando aquellas vinculadas al egoísmo, los sesgos cognitivos y la racionalidad de los individuos en sus tomas de decisiones, entendiendo por sesgos cognitivos aquellas reglas heurísticas que moldean las preferencias y decisiones de los individuos de forma que se enfrenta a la racionalidad humana (Moynihan y Lavertu, 2012).

En esta línea, un creciente número de investigaciones indica que los generadores de la utilidad no son estados, sino cambios relativos respecto de un punto de referencia. Por ello, se desarrolla un modelo de comportamiento en base al que cuestionar los planteamientos clásicos de elección del agente para el caso de que éste venga disfrutando de algún bien o servicio existente en el mercado. En este caso, en el denominado modelo conductual de elección, los individuos podrían presentar una tendencia a preferir la opción actual, también denominada decisión en uso, independientemente de la existencia de una alternativa que presente una utilidad superior. Ello se debe a que, cuando los consumidores han optado por una opción, sus preferencias son asimétricas debido a la tendencia a mantenerse en el estado actual (Kahneman et al., 1991; Wang et al., 2013).

Las políticas públicas necesitan comprender mejor el comportamiento humano y promover su cambio a través de un enfoque más científico, habiéndose destacado los hallazgos conductuales como fórmula para la creación de políticas públicas de un «nivel superior» (OECD, 2017). Los programas públicos exitosos descansan en un ajuste a la manera en la que los ciudadanos se comportan y eligen. La economía conductual, al modificar la forma en la que se piensa acerca de por qué se elige de una determinada forma y qué motiva tales decisiones, permitiendo entender el comportamiento de los ciudadanos. Esto viene a complementar los hallazgos de la economía neoclásica vinculados a las asimetrías de la información y ayuda a comprender tanto por qué fallan los programas públicos como qué se puede hacer (Datta y Mullainathan, 2014). El uso de los hallazgos conductuales implica un cambio de paradigma en la forma en la que la administración pública trabaja, partiendo de lo que funciona a nivel micro para determinar su aplicabilidad a una mayor escala (OECD, 2015).

En la administración pública conductual el conocimiento generado a través de la observación y experimentación se emplea para identificar pautas de comportamiento y emplearlas en el diseño de las políticas y regulaciones. Se opta por un enfoque inductivo en el que el conocimiento generado viene a reemplazar lo que se considera comportamiento tradicional de los ciudadanos. La demanda de intervenciones gubernamentales más efectivas y eficientes dotan de relevancia la aplicación de los hallazgos conductuales en la política pública (OECD, 2017). Pese a lo expuesto, ha existido un limitado esfuerzo por comprender las implicaciones de los sesgos cognitivos sobre las preferencias, decisiones y acciones de los agentes públicos (Moynihan y Lavertu, 2012), reclamándose una mayor aplicación de los principios conductuales al sector público (Choueiki, 2016).

Una teoría empleada para explicar la forma en la que el uso de un sistema de información (SI) impacta las percepciones e intenciones vinculadas a uno nuevo es la tendencia al statu quo (SQB), que analiza el impacto que supone mantener la situación actual traducida en percepciones inhibidoras del uso de un nuevo SI (Hsieh, 2016; Kahneman et al., 1991). La SQB se apoya en argumentaciones teóricas, dependientes del contexto, relativas a los motivos por los que los individuos toman decisiones que los llevan a mantener un determinado curso de acción, más que cambiar a un nuevo, aunque pueda llegar a ser potencialmente superior (Polites y Karahanna, 2012; Wang et al., 2013). Resulta, por ello, fundamental para los proveedores de servicios digitales identificar los factores que podrían fortalecer o debilitar el statu quo, ya que podrían desarrollar estrategias para reforzar la intención de los ciudadanos de cambiar entre los diferentes canales digitales para acceder a determinados servicios (Li y Cheng, 2014). 


\subsection{Las campañas públicas de cambio de comportamiento}

La modificación del comportamiento de los ciudadanos respecto del canal empleado para acceder a los servicios públicos se vincula a las estrategias de migración, que pueden ser divididas en dos: estrategias centradas en el diseño, que determinan el orden de migración dentro de la estrategia multicanal; y las estrategias que guían el proceso de implantación, que conducen la implantación del proyecto, no centrándose en como diseñar la estrategia multicanal sino en el proceso de migración (van Veenstra y Janssen, 2010). Debido a que la respuesta vinculada al hábito es rápida de activar en la memoria, y el rastro de la memoria habitual es lento de cambiar en base a nuevas experiencias, son necesarios esfuerzos cognitivos y motivacionales para lograr que el ciudadano responda de una forma que contravenga los hábitos. Para actuar de una forma no habitual, deben tomar la decisión de hacer algo nuevo y, adicionalmente, anular la respuesta habitual accesible en la memoria. Los hábitos tienen un doble filtro en el trabajo de la memoria y en otros recursos cognitivos. Dada la naturaleza intensiva en recursos de decidir y anular, los ciudadanos son proclives a actuar de forma habitual cuando se exponen a las presiones de tiempo, las distracciones y las regulaciones del día a día que reducen sus recursos cognitivos (Neal y Wood, 2006).

Desde la administración pública se desarrollan campañas con el objetivo de cambiar el comportamiento de los ciudadanos en base a la modificación de sus hábitos. Dicho cambio de hábitos resulta complejo porque su formación se vincula con el desarrollo de expectativas acerca del comportamiento y del efecto del entorno. Tales expectativas basadas en la repetición reducen la sensibilidad ante variaciones mínimas en el entorno, limitando tanto la búsqueda de información como el pensamiento y deliberación acerca de la acción. Los ciudadanos con fuertes hábitos desarrollan expectativas para entornos y comportamientos concretos. Las personas con fuertes hábitos esperan que se repitan las expectativas previas y no detectan fácilmente cambios menores en el entorno. Se señalan dos elementos clave en el vínculo entre hábito y comportamiento que llevan a que los ciudadanos empleen menos información y su comportamiento esté menos guiado por las actitudes e intenciones (Maio et al., 2007; Verplanken y Wood, 2006): (1) realizan búsquedas menos extensas acerca de las alternativas existentes que tienden a sesgarse hacia la confirmación de lo habitual, ya que las personas con fuertes hábitos poseen sesgos que reducen la posibilidad de que reciban y evalúen favorablemente información en sentido opuesto; y, (2) los hábitos fuertes se asocian con reglas de decisión simples y limitadas, de forma que el comportamiento basado en el hábito está menos guiado por las actitudes e intenciones que aquel conducido de una forma más pensada y deliberada.

Que el hábito se vincule con entornos recurrentes provoca que cambios en los elementos del entorno abran la posibilidad a cambios en los hábitos, identificándose inicialmente dos tipos de actuaciones para modificar los hábitos de los ciudadanos (Verplanken y Wood, 2006). Las intervenciones descendentes, centradas en modificar el comportamiento cuando existe un factor significativo de riesgo. Tratan el problema a través del propio interesado y se aplican a comportamientos que podrían evolucionar a hábitos con el tiempo. Un enfoque descendente clásico es buscar el cambio del comportamiento a través de campañas de información. Un elemento clave vinculado a estas campañas es el sentimiento de ambivalencia hacia el comportamiento recomendado. Por contra, las intervenciones ascendentes se centran en cambiar el entorno en el que se desarrolla el comportamiento problemático y promover alternativas diseñadas para prevenir comportamientos no deseados. Estas intervenciones ascendentes se focalizan en las normas sociales y en el apoyo del entorno para el comportamiento deseado. Las intervenciones ascendentes tratan de adaptar las condiciones que pueden promover y sostener el hábito deseado pudiendo ser difíciles de llevar a cabo, involucrando recursos sustanciales a lo largo de prolongados periodos de tiempo, así como apoyo político. Para resultar exitosas debe darse una combinación de legislación, refuerzo, educación y cambio estructural (Maio et al., 2007).

En tercer lugar, se exponen las intervenciones combinadas. Si el hábito presenta barreras al cambio, se podrían buscar aquellas situaciones donde los hábitos pueden ser modificados temporalmente. Verplanken y Wood (2006) las denominan intervenciones descendentes con cambios en el entorno, y su supuesto de partida es que los individuos se encuentren en una posición que requiera encontrar nuevas formas para comportarse, resultando más susceptibles a aquello que guía nuevos comportamientos (Maio et al., 2007). Estas intervenciones se vinculan a comportamientos no deseados con un fuerte componente habitual, a cambios en los estilos de vida. De forma específica, aquellos cambios en el entorno que modifican los hábitos también pueden suponer un reto para los esquemas mentales de los ciudadanos e incrementar la apertura a nuevos experimentos e informaciones. Debido a que tales cambios en el entorno distorsionan las respuestas automáticas, permiten el desarrollo de nuevas acciones. Algunos autores han señalado el papel de los eventos diarios como una ventana de oportunidad para el cambio (Schäfer et al., 2012). 
En otras ocasiones se sugiere la existencia de dos tipos de intervenciones para conseguir cambios en el comportamiento: intervenciones reductoras, que transforman los hábitos reforzando la autorregulación y suministrando información, e intervenciones acrecentadoras que interrumpen los entornos y establecen diferentes elementos que facilitan la formación de nuevos hábitos. La magnitud que el efecto de una intervención tiene sobre los consumidores depende de si perciben su entorno como relativamente estable. Es por ello que la forma en la que el consumidor procesa la información generada por la intervención puede moderar su efecto sobre el cambio de comportamiento. En un contexto multicanal, los consumidores tienden a persistir en su rutina habitual si perciben el uso del nuevo canal como la evolución natural de la continuación de su relación con la organización (Wang et al., 2015).

\section{MÉTODOS}

\subsection{Modelo de investigación e hipótesis}

Ya que el presente trabajo analiza el impacto que determinados sesgos cognitivos (hábito, inercia y resistencia) presentan sobre la intención (de uso o continuación) de los ciudadanos en relación al e-gobierno, pasamos a realizar una delimitación de tales elementos.

Triandis (1980) define el hábito como una secuencia de comportamientos vinculados a una situación que se han convertido en automáticos. En otras ocasiones, se define como una secuencia aprendida de actos originalmente intencional convertida en respuestas a asuntos específicos y funcionales en el logro de determinados objetivos, secuencia que podría haber sido aprendida sin una intención consciente bajo unas condiciones estables del contexto. Cuando el uso de las TIC es habitual, puede no estar guiado por una planificación consciente y verse condicionado por el entorno sin pensarlo o de forma automática (Ortiz de Guinea y Markus, 2009; Polites y Karahanna, 2012). Para que un comportamiento repetido pueda ser tildado de hábito, éste debe darse con cierto grado de automaticidad (Olsen, et al., 2013; Verplanken y Aarts, 1999; Verplanken y Wood, 2006). La automaticidad vinculada a muchas actividades cotidianas, así como el carácter estable del contexto, hacen difícil motivar a las personas a cambiar sus pautas de comportamiento (Schäfer et al., 2012).

Continuando con los sesgos cognitivos, la inercia es una emoción inconsciente que puede ser guiada por la conveniencia, un uso repetido de algo conocido desarrollado de forma pasiva sin pensar en ello y sin tener presentes las percepciones negativas que se le vinculan. La inercia refleja la carencia de un deseo por abandonar el statu quo independientemente de las alternativas presentes o de aquellas que pudieran aparecer, describe una tendencia de comportamiento que lleva a descansar en lo elegido anteriormente y en lo que representa el estado actual. Es por ello que la inercia se define como una secuencia aprendida de actos que convertidos en respuestas automáticas ante asuntos específicos resultan funcionales (Verplanken y Aarts, 1999). La inercia afecta a la disposición de los consumidores a probar e-servicios o nuevas tecnologías (Falk et al., 2007)

Dentro de los factores inconscientes que condicionan el comportamiento presenta una especial relevancia la resistencia, definida como el rechazo ofrecido por las personas, tanto porque supongan cambios en un statu quo satisfactorio como un conflicto con su estructura de creencias (Patsiotis et al., 2013). La resistencia se refiere a una fuerza que dificulta la innovación, que en el ámbito de los SI se define como la oposición -o reacción adversa- de un usuario hacia el cambio vinculado con su implantación (Kim y Kankanhalli, 2009). En su aplicación al e-gobierno, se la define como la medida en la que los servicios de gobierno electrónico son obstruidos o dilatados (Ebbers y van Dijk, 2007).

Mientras la intención de uso se refiere a la intención manifestada de desarrollar un determinado comportamiento, la continuación -o uso continuado- es aquel comportamiento vinculado a una fase posterior a la aceptación. En la continuación, el uso del SI trasciende el comportamiento consciente y pasa a formar parte de una actividad rutinaria normal. La decisión de continuar (1) sigue una decisión inicial, (2) es influenciada por el uso inicial y (3) puede llevar a revertir una decisión inicial (Bhattacherjee, 2001). La continuación en el uso se refiere a la tendencia a continuar con un comportamiento y mantener el statu quo (Khedhaouria et al., 2016).

El uso habitual de un servicio es una de las principales motivaciones del comportamiento del consumidor. Cuando se comienza a desarrollar una acción, las personas deciden qué hacer y cómo hacerlo para alcanzar determinados resultados. La formación de hábitos supone crear asociaciones en la memoria entre 
las acciones y los rasgos estables del entorno en que se desarrollan. Dicha estabilidad del entorno lleva a que las respuestas de las personas se realicen directamente sin necesitar de ningún elemento relativo a la intención o decisión de actuar (Neal y Wood, 2006, Verplanken y Wood, 2006). Relevancia del entorno que recientemente ha sido verificada para el caso de la prestación digital de servicios públicos (Castelnovo y Sorrentino, 2017). El hábito se constituye en una fuente inconsciente de inercia (Ng y Kwahk, 2010; Polites y Karahanna, 2012), afirmándose que los consumidores continúan haciendo algo debido a la inercia resultante del hábito (Khedhaouria et al., 2016). La resistencia viene determinada por el compromiso psicológico, por la toma de decisión racional y por la ausencia de percepción cognitiva, entre cuyos componentes se encuentra la inercia, considerándose que la inercia tiene un efecto positivo en la resistencia (Hsieh, 2016; Hsieh y Lin, 2017). Es por lo expuesto que planteamos las siguientes hipótesis:

- H1: el hábito se vincula positivamente con la inercia desarrollada hacia el SI.

- H2: la inercia se vincula positivamente con la resistencia respecto al uso del SI.

Entre los determinantes de la intención de continuación de un SI se han identificado el hábito, la inercia y la resistencia (Khedhaouria et al., 2016; Kim y Gupta, 2012; Hsieh, 2016; Hsieh y Lin, 2017). Los individuos guiados por la inercia evitan la búsqueda de la variedad, pudiendo convertirse en una aversión a la innovación, incluso ante mejores alternativas. Emplear el canal online por primera vez supone un cambio de comportamiento en el que las pautas adquiridas deben ser alteradas. La inercia influye negativamente en la disposición a interactuar con tecnologías innovadoras (Falk et al., 2007). La inercia es un determinante inconsciente de la intención de continuar, una manifestación de la tendencia al statu quo (Khedhaouria et al., 2016; Kim y Gupta, 2012). No obstante, en ocasiones se indica que la relación entre inercia e intención de uso es inversa (Hsieh, 2016; Hsieh y Lin, 2017). La resistencia puede presentar diversos resultados, entre ellos una mayor búsqueda de información, posponer el uso o bien oponerse a la innovación (Patsiotis et al., 2013). Por estas razones, se llega a establecer que la resistencia se constituye en el primer reto para la implementación de los SI, incluso como canales de distribución (Kim y Kankanhalli, 2009). Falk et al. (2007) parten de que el canal tradicionalmente empleado puede ser considerado como una alternativa «en posesión», mientras que el canal online constituye una «nueva alternativa» dentro del conjunto en comparación. Pudiéndose establecer las siguientes hipótesis:

- H3: el hábito se vincula positivamente con la intención de comportamiento al e-gobierno.

- H4: la inercia se vincula positivamente con la intención de comportamiento respecto al e-gobierno.

- H5: la resistencia se vincula positivamente con la intención de comportamiento respecto al e-gobierno.

Conforme el hábito se desarrolla y gana fuerza, la planificación que se lleva a cabo es menor, y la lealtad se ve guiada, principalmente, por la automaticidad y la inercia. Convirtiéndose la inercia en una variable mediadora entre el hábito y el comportamiento (Olsen et al., 2013). Una fuente potencial de resistencia en la adopción de un nuevo SI se encuentra en el hábito hacia el SI en uso. Cuando los individuos adquieren hábitos a través de un uso rutinario a lo largo del tiempo, desarrollan comportamientos automáticos que en ocasiones sirven para mantener el statu quo (Falk et al., 2007; Ng y Kwahk, 2010; Polites y Karahanna, 2012). Ya que la implantación de un nuevo SI supone reemplazar el sistema actualmente empleado, los usuarios se podrían formar expectativas basadas en el conocimiento y conforme se habitúan, tal uso supone un fuerte anclaje para evaluar el nuevo sistema que podría basarse en un reducido volumen de información obtenida de sus experiencias recientes y formar sus expectativas sobre el nuevo sistema basándose en la información ofrecida por la organización (Lee y Joshi, 2016). Dado que la adopción de un nuevo SI implica reemplazar un sistema por otro, la resistencia se manifiesta como la reticencia a cambiar a una nueva tecnología. A menudo, una fuente potencial de resistencia parte del uso del SI que se está empleando, vinculándose con el hábito (Patsiotis et al., 2013; Polites y Karahanna, 2012). En esta línea Polites y Karahanna (2012) proponen que la inercia media entre el impacto del hábito del sistema actual y los costes de cambio percibidos sobre las creencias e intenciones vinculadas al nuevo sistema, esto es, sobre la intención de comportamiento. De forma más explícita, para Khedhaouria et al. (2016) la inercia media el impacto que sobre la intención de continuar presenta el hábito. Es por ello, que se pueden establecer las siguientes hipótesis:

- H6: el hábito se relaciona, de forma mediada, con la resistencia al e-gobierno.

- H7: el hábito se relaciona, de forma mediada, con la intención de comportamiento al e-gobierno.

- H8: la inercia se relaciona, de forma mediada, con la intención de comportamiento al e-gobierno. 


\subsection{Medición de las variables}

Las variables, ítems empleados para medirlas, así como los trabajos de los que proceden aparecen en la tabla siguiente (tabla 3 ).

TABLA 3. VARIABLES ANALIZAdAS (AUTORES), ÍTEMS Y ACRÓNIMOS

\begin{tabular}{|c|c|c|}
\hline $\begin{array}{c}\text { Variables analizadas } \\
\text { (fuentes) }\end{array}$ & ítems & Acrónimo \\
\hline \multirow{3}{*}{$\begin{array}{l}\text { Resistencia } \\
\text { (Bhattacherjee } \\
\text { y Hikmet, 2007; } \\
\text { Hsieh, 2016) }\end{array}$} & $\begin{array}{l}\text { No me gusta que la Administración Electrónica cambie la forma en que acce- } \\
\text { do a los servicios públicos. }\end{array}$ & RE1 \\
\hline & $\begin{array}{l}\text { No me gusta que la Administración Electrónica cambie la forma en que tomo } \\
\text { decisiones relativas al uso los servicios públicos. }\end{array}$ & RE2 \\
\hline & $\begin{array}{l}\text { No me gusta dejar de tener contacto personal con los funcionarios al realizar } \\
\text { mis gestiones. }\end{array}$ & RE3 \\
\hline \multirow{3}{*}{$\begin{array}{l}\text { Inercia (Khedhaouria } \\
\text { et al., 2016; Polites } \\
\text { y Karahanna, 2012) }\end{array}$} & $\begin{array}{l}\text { Continuaré empleando el canal de comunicación actual con la Administración } \\
\text { porque me estresa cambiarlo. }\end{array}$ & IN1 \\
\hline & $\begin{array}{l}\text { Continuaré empleando el canal de comunicación actual con la Administración } \\
\text { porque disfruto empleándolo. }\end{array}$ & IN2 \\
\hline & $\begin{array}{l}\text { Continuaré empleando el canal de comunicación actual, incluso aunque sepa } \\
\text { que no es la mejor forma de hacer las cosas. }\end{array}$ & IN3 \\
\hline \multirow{3}{*}{$\begin{array}{l}\text { Hábito (Khedhaouri } \\
\text { et al., 2016; Limayen } \\
\text { et al., 2007) }\end{array}$} & Emplear el canal que actualmente empleo es algo natural para mí. & HA1 \\
\hline & $\begin{array}{l}\text { Después de haberlo utilizado varias veces, hacer gestiones con la Administra- } \\
\text { ción a través del canal que empleo actualmente resulta bastante automático. }\end{array}$ & HA2 \\
\hline & $\begin{array}{l}\text { Cuando tengo varias opciones para realizar una gestión prefiero hacerla a } \\
\text { través del canal que empleo actualmente. }\end{array}$ & HA3 \\
\hline \multirow{3}{*}{$\begin{array}{l}\text { Intención de uso } \\
\text { (Taylor y Todd, 1995) }\end{array}$} & Tengo la intención de utilizar la Administración Electrónica. & IU1 \\
\hline & Tengo la intención de utilizar algunos de los servicios. & IU2 \\
\hline & $\begin{array}{l}\text { Mi intención es ir haciendo cada vez más gestiones a través de la Administra- } \\
\text { ción Electrónica. }\end{array}$ & IU3 \\
\hline \multirow{2}{*}{$\begin{array}{l}\text { Intención de } \\
\text { continuación } \\
\text { (Venkatesh et al., 2011). }\end{array}$} & El uso de la Administración Electrónica es ya un hábito para mí. & IC1 \\
\hline & Utilizaré la Administración Electrónica siempre que exista esa posibilidad. & IC2 \\
\hline
\end{tabular}

Fuente: elaboración propia.

Como se puede observar, se empleó en el cuestionario el término administración electrónica en lugar de e-gobierno. Al inicio de la entrevista se realizaba la siguiente aclaración a los encuestados "La administración electrónica se refiere a la posibilidad que los ciudadanos y empresas tienen de relacionarse con las instituciones y organismos del estado, de las comunidades autónomas y de los ayuntamientos, utilizando las nuevas tecnologías de la información y la comunicación, para realizar de forma telemática a través de internet consultas y gestiones de todo tipo que antes tenía que hacer personalmente o por teléfono (por ejemplo pedir información, presentar quejas, entregar documentación, solicitar cita, entregar declaraciones de impuestos, hacer la declaración de la renta, pagar el IBI, el IAE, etc.)». De este modo, aunque se indica administración electrónica, se refiere a la conceptualización del e-gobierno indicada en la introducción del presente trabajo, sin centrarse en ningún nivel administrativo ni ámbito competencial.

\subsection{Muestra y datos}

Para la verificación de la hipótesis se empleó una metodología cualitativa y cuantitativa. La cualitativa se desarrolló mediante dos grupos de discusión de no-usuarios y usuarios del e-gobierno. El grupo de los usuarios regulares del e-gobierno estaba compuesto por un total de 7 personas (4 hombres y 3 mujeres) entre los 26 y los 59 años y tuvo una duración de 71 minutos. El grupo de los no usuarios del e-gobierno estuvo compuesto por 6 participantes ( 3 hombres y 3 mujeres) entre los 37 y los 59 años y tuvo una duración de 83 minutos. Se estableció el criterio diferenciador del uso del gobierno electrónico ya que dicha diferencia ge- 
nera discursos contrapuestos en los que se pueden identificar con facilidad los motores y frenos para el uso de las vías telemáticas de la administración. Con el desarrollo de los grupos se verificó la relevancia de las variables objeto de análisis y confirmó la relevancia de los factores facilitadores e inhibidores contemplados, así como para adaptar las escalas en el trabajo cuantitativo.

En el trabajo de campo se captaron 923 cuestionarios, 541 correspondientes a usuarios del e-gobierno y 382 correspondientes a no usuarios del e-gobierno. En la siguiente tabla se presenta el perfil de la muestra.

TABLA 4. PERFIL DE LA MUESTRA

\begin{tabular}{|c|c|c|c|c|}
\hline Variable & Rangos & Total & Usuarios & No usuarios \\
\hline \multirow[t]{4}{*}{ Edad } & De 18 a 29 años & 82 & 54 & 28 \\
\hline & De 30 a 44 años & 284 & 203 & 81 \\
\hline & De 45 a 64 años & 456 & 252 & 204 \\
\hline & De 65 y más años & 101 & 32 & 69 \\
\hline \multirow[t]{2}{*}{ Género } & Hombre & 385 & 231 & 154 \\
\hline & Mujer & 538 & 310 & 228 \\
\hline \multirow[t]{6}{*}{ Nivel de estudios } & BUP, Bachiller Superior y FP2 & 260 & 152 & 108 \\
\hline & EGB, Bachiller Elemental y FP1 & 182 & 71 & 111 \\
\hline & Estudios Medios (Peritaje, ATS, etc) & 114 & 74 & 40 \\
\hline & Estudios Superiores & 301 & 224 & 77 \\
\hline & NS/NC & 12 & 5 & 7 \\
\hline & Sin Estudios y primarios & 54 & 15 & 39 \\
\hline
\end{tabular}

Como se puede observar, un $80 \%$ de la muestra se encuentra en la franja de edad entre 30 y 64 años. Mientras que en la muestra de usuarios del e-gobierno la franja entre 30 y 44 años supone un $38 \%$, se reduce a un $21 \%$ en la muestra de no usuarios. En el caso del género la muestra total está compuesta por un $42 \%$ de hombres y un $58 \%$ de mujeres, cifras que no sufren grandes modificaciones para la muestra de usuarios y de no usuarios. Respecto al nivel de estudios, la representación del segmento «EGB, Bachiller Elemental y FP1» es del $13 \%$ en los usuarios y del $29 \%$ para los no usuarios. Los usuarios con «Estudios Superiores» es del $41 \%$ en los usuarios y del $20 \%$ para los no usuarios.

Debido a que las escalas empleadas responden a la adaptación de escalas validadas en trabajos previos, asumimos su validez de contenido. El análisis estadístico se realizó mediante una modelización de ecuaciones estructurales (SEM) aplicando la técnica Partial Least Squares (PLS) con SmartPLS 3.2.6. Conceptualmente, PLS resulta muy similar al uso del análisis de la regresión múltiple. Su primer objetivo en maximizar la varianza explicada de la variable dependiente, al tiempo que evalúa la calidad de los datos en base a las características del modelo de medida. PLSSEM emplea un algoritmo en dos etapas. En la primera se estiman los scores de los constructos latentes de forma iterativa, en la segunda calcula las estimaciones finales de los coeficientes (pesos, cargas y coeficientes) empleando el método de los Mínimos Cuadrados Ordinarios para cada regresión parcial del modelo (Hair et al., 2011). Aplicando ambos procesos, busca minimizar la varianza residual de cada variable dependiente y, ya que PLSSEM no realiza ninguna asunción acerca de la especificación de la predicción de las relaciones causa-efecto entre las variables, deben ser definidas de modo específico (Roldán y Sánchez-Franco, 2012). Se diferencian dos etapas en el análisis PLS, la evaluación del modelo de medida (define las variables latentes que emplea el modelo y le asigna los indicadores a cada una, tratando de analizar si los constructos teóricos son representados de forma correcta por las medidas) y la evaluación del modelo estructural (define las relaciones entre las variables latentes).

\subsection{Análisis del modelo de medida}

El modelo de medida incluye las relaciones unidireccionales predictivas entre cada constructo latente y los indicadores observados asociados al mismo. En el modelo correspondiente a los no-usuarios del egobierno, se debió eliminar el ítem HA2, al que se le une, en el modelo correspondiente a los usuarios los 
ítems IN1 e IN3. Como medida de la fiabilidad interna se emplea el índice de fiabilidad compuesta. Se sugiere que 0,7 es un valor de fiabilidad aceptable en las etapas iniciales de investigación, y que unos niveles más estrictos de 0,8 lo son para etapas avanzadas. Como se puede observar en la tabla inferior (tabla 5), todos los constructos superan dicho criterio más estricto.

TABLA 5. FIABILIDAD COMPUESTA INTERNA

\begin{tabular}{lccc}
\hline & Constructo & No-Usuarios & Usuarios \\
\hline $\mathrm{HA}$ & 0,902 & 0,804 \\
\hline $\mathrm{IN}$ & 0,813 & 1,000 \\
\hline $\mathrm{IU} / \mathrm{IC}$ & 0,918 & 0,894 \\
\hline $\mathrm{RE}$ & 0,836 & 0,797 \\
\hline
\end{tabular}

Fuente: elaboración propia.

La validez convergente indica que un mismo conjunto de indicadores representan al mismo constructo subyacente, y, en primer lugar, se establece a través del análisis de la Average Variance Extracted (AVE), planteándose que sus valores deben ser superiores a 0,5 , valor superado en los modelos. Asimismo, los constructos cumplen con el criterio VIF, aceptándose la validez convergente de los constructos empleados (tabla 6).

TABLA 6. FIABILIDAD DE LOS CONSTRUCTOS

\begin{tabular}{lcccccc}
\hline \multicolumn{1}{c}{ Constructo } & AVE & \multicolumn{5}{c}{ VIF (No-usuarios/Usuarios) } \\
\hline & No-usuarios & Usuarios & HA & IN & IU/IC & RE \\
\hline HA & 0,821 & 0,673 & $-/-$ & $1,000 / 1,000$ & $1,394 / 1,198$ & $-/-$ \\
\hline IN & 0,592 & 1,000 & $-/-$ & $-/-$ & $1,415 / 1,196$ & $1.000 / 1,000$ \\
\hline IU/IC & 0,788 & 0,809 & $-/-$ & $-/-$ & $-/-$ & $-/-$ \\
\hline RE & 0,629 & 0,568 & $-/-$ & $-/-$ & $1,068 / 1,004$ & $-/-$ \\
\hline
\end{tabular}

Fuente: elaboración propia.

La validez discriminante indica el grado en el que un constructo difiere de otros empleándose para su confirmación dos criterios. De acuerdo con el primero, el valor AVE debe ser superior a la varianza compartida entre el constructo y los demás constructos representados. Los constructos analizados superan dicho criterio (tabla 7).

TABLA 7. VALIDEZ DISCRIMINANTE I. CARGAS CRUZADAS (NO-USUARIOS/USUARIOS)

\begin{tabular}{lcccc}
\hline & HA & IN & IU/IC & RE \\
\hline HA & $0,906 / 0,820$ & $-/-$ & $-/-$ & $-/-$ \\
\hline IN & $0,526 / 0,404$ & $0,769 / 1,000$ & $-/-$ & $-/-$ \\
\hline IU/IC & $-0,301 / 0,319$ & $-0,338 / 0,165$ & $0,888 / 0,899$ & $-/-$ \\
\hline RE & $0,202 /-0,061$ & $0,235 /-0,039$ & $-0,240 /-0,230$ & $0,793 / 0,753$ \\
\hline
\end{tabular}

Como segundo criterio de la validez discriminante, se empleó el hetero-trait-monotrait (HTMT) ratio de correlaciones. Para la muestra tanto de los usuarios de la administración telefónica como de la presencial, todos los constructos superan el criterio conservador HTMT.85.

TABLA 8. VALIDEZ DISCRIMINANTE II. CRITERIO HTMT (NO-USUARIOS/USUARIOS)

\begin{tabular}{lllll}
\hline & HA & IN & IU/IC & RE \\
\hline HA & $-/-$ & $-/-$ & $-/ 0,500$ & $-/-$ \\
\hline IN & $0,731 / 0,559$ & $-/-$ & $-/ 0,188$ & $-/-$ \\
\hline IU/IC & $0,366 /-$ & $0,448 /-$ & $-/-$ & $-/-$ \\
\hline RE & $0,251 / 0,109$ & $0,319 / 0,047$ & $0,286 / 0,310$ & $-/-$ \\
\hline
\end{tabular}




\subsection{Limitaciones}

Como principal limitación del presente trabajo, se debe tener presente que el modelo se compone de relaciones mediadas y que los constructos incorporados en las mismas deberían ser medidos en diferentes momentos del tiempo. Asimismo, se deberían incorporar en el modelo elementos que permitan analizar el vínculo entre hábito y resistencia para los usuarios del e-gobierno.

\section{RESULTADOS}

\subsection{Análisis del modelo estructural}

En el modelo estructural se diferencia entre constructos exógenos y endógenos. El término exógeno describe aquellos constructos latentes que no son alcanzados por ningún path estructural. Por su parte, el término endógeno describe los constructos latentes del modelo estructural que son explicados por otros constructos vía relaciones estructurales. PLS sólo permite relaciones recursivas en el modelo estructural, es decir, los path entre los constructos latentes sólo pueden tener una dirección (Hair et al., 2011). El primer criterio a emplear es el coeficiente de determinación de los constructos endógenos $\left(R^{2}\right)$, que representa una medida del poder predictivo e indica la cantidad de varianza del constructo en cuestión que es explicada por sus variables antecedentes en el modelo. Se recomienda un valor para $R^{2}$ superior a 0,10 . Asimismo, el test de Stone-Geiser (Q2) es empleado para evaluar la relevancia predictiva de los constructos con una medida reflectiva, considerándose que, si toma un valor superior a 0 para una variable endógena, el modelo presenta capacidad predictiva. De acuerdo con los resultados obtenidos en los modelos (véase tabla 9), se puede aceptar la capacidad predictiva de los modelos para las variables endógenas inercia e intención, de uso o continuación en cada caso.

TABLA 9. EVALUACIÓN MODELO ESTRUCTURAL

\begin{tabular}{lcccc}
\hline \multirow{2}{*}{ Constructo } & \multicolumn{2}{c}{$\mathbf{R}^{2}$} & \multicolumn{2}{c}{$\mathbf{Q}^{2}$} \\
\cline { 2 - 5 } & No-usuarios & Usuarios & No-usuarios & Usuarios \\
\hline $\mathrm{HA}$ & - & - & - & - \\
\hline $\mathrm{IN}$ & 0,276 & 0,164 & 0,153 & 0,157 \\
\hline $\mathrm{IU} / \mathrm{IC}$ & 0,158 & 0,148 & 0,115 & 0,108 \\
\hline $\mathrm{RE}$ & 0,055 & 0,002 & 0,027 & $-0,000$ \\
\hline & Fuente: elaboración propia. & &
\end{tabular}

A continuación, se analizan los path, o coeficientes de regresión estandarizados estimados, debiéndose tenerse presente el signo, magnitud y significación ${ }^{4}$. Aquellos path $(\beta)$ significativos y cuyo signo sigue la dirección marcada en la hipótesis apoyan la relación propuesta.

Para aquellos ciudadanos no usuarios del e-gobierno el hábito presenta una relación directa significativa sobre la inercia $(\beta=0,526, t=10,741)$ y sobre intención de uso del e-gobierno $(\beta=-0,153, t=2,747)$. La inercia, se relaciona significativamente con la resistencia $(\beta=0,235, t=4,228)$ y con la intención de uso $(\beta=-0,220$, $t=3,931$ ), finalmente, la resistencia se relaciona de forma significativa con la intención de uso del e-gobierno $(\beta=-0,157, t=2,671)$. Para los usuarios del e-gobierno, el hábito presenta una relación directa significativa sobre la inercia $(\beta=0,404, t=9,409)$ y sobre intención de continuación de uso del e-gobierno $(\beta=0,040$, $t=6,106)$. No resulta significativa la relación directa de la inercia con la resistencia $(\beta=-0,039, t=0,831)$ ni con la intención de continuación en el uso $(\beta=-0,220, t=0,869)$, finalmente, la resistencia se relaciona de forma significativa con la intención de continuación de uso del e-gobierno $(\beta=-0,210, t=5,175)$.

En el caso de las relaciones mediadas, para la muestra de no-usuarios del e-gobierno, resulta significativa la relación del hábito con la intención de uso $(\beta=-0,135, t=4,181)$ y con la resistencia $(\beta=0,123$, $t=3,688)$, y la de la inercia con la intención de uso $(\beta=-0,037, t=2,309)$. Para la muestra de los usuarios del e-gobierno, ninguna relación mediada resulta significativa, ni la de hábito con intención de continuación

${ }^{4}{ }^{*} \mathrm{p}, 0,05 ;{ }^{* *} \mathrm{p}, 0,01 ;{ }^{* *} \mathrm{p}, 0,001$ basada en una distribución t de Student de una cola $(499), \mathrm{t}(0,05 ; 499)=1,6479 ; \mathrm{t}(0,01 ;$ $499)=2,3338 ; \mathrm{t}(0,001 ; 499)=3,1066$. 
$(\beta=0,019, t=1,009)$ o resistencia $(\beta=-0,016, t=0,814)$, ni la de la inercia con la intención de continuación $(\beta=0,008, t=0,802)$.

\subsection{Análisis de los resultados}

Las escalas empleadas resultan adecuadas, salvo para el caso de la inercia, ya que se ha debido eliminar dos de los tres ítems empleados para su medición en la muestra correspondiente a los usuarios del e-gobierno. No obstante, como ha quedado establecido a través de la fiabilidad compuesta a través del AVE y del criterio HTMT.85, el modelo de medida respeta los niveles requeridos.

En el caso del modelo estructural, se debe tener presente que el modelo no presenta capacidad predictiva en relación a la resistencia, ya que presenta bajos valores para la muestra de los no-usuarios $\left(R^{2}\right.$ nousuarios $=0,055, Q^{2}$ no-usuarios $=0,002$ ) y no supera el nivel requerido para el test de Stone Geiser en el caso de la muestra de usuarios del e-gobierno $\left(R^{2}\right.$ usuarios $=0,027, Q^{2}$ usuarios $\left.=-0,000\right)$.

En el caso de la muestra de no-usuarios del e-gobierno, quedan verificadas todas las relaciones planteadas en el modelo, tanto las directas como las mediadas, por lo que se puede aceptar que el modelo planeado explica la intención de uso del e-gobierno.

Para el caso de los usuarios del e-gobierno, no resultan significativas las relaciones que parten de la inercia, de forma que el hábito determina la inercia y la intención de continuación, que se ve determinada también por la resistencia. En este modelo, ninguna de las relaciones mediadas resulta significativa.

TABLA 10. RESUMEN DE LAS HIPÓTESIS

\begin{tabular}{|c|c|c|c|}
\hline Hipótesis & No-usuarios & Usuarios & Resultado \\
\hline $\mathrm{H} 1$ & Significativa & Significativa & Aceptada \\
\hline $\mathrm{H} 2$ & Significativa & No significativa & No aceptada \\
\hline $\mathrm{H} 3$ & Significativa & Significativa & Aceptada \\
\hline $\mathrm{H} 4$ & Significativa & No significativa & No aceptada \\
\hline H5 & Significativa & Significativa & Aceptada \\
\hline $\mathrm{H} 6$ & Significativa & No significativa & No aceptada \\
\hline $\mathrm{H} 7$ & Significativa & No significativa & No aceptada \\
\hline H8 & Significativa & No significativa & No aceptada \\
\hline
\end{tabular}

La relación del hábito con la inercia y la intención de comportamiento resulta significativa para los nousuarios y usuarios del e-gobierno, por lo que se pueden aceptar $\mathrm{H} 1$ y H3. Asimismo, resulta significativa para ambas muestras la relación entre resistencia e intención de comportamiento aceptándose $\mathrm{H} 5$. Al no resultar las relaciones para la muestra de usuarios del e-gobierno, no se pueden aceptar las restantes relaciones ( $\mathrm{H} 2, \mathrm{H} 4, \mathrm{H} 5, \mathrm{H} 6, \mathrm{H} 7$ y $\mathrm{H} 8)$.

\section{DISCUSIÓN DE LOS RESULTADOS}

El presente trabajo se ha desarrollado con el objetivo de profundizar en el conocimiento de los elementos que determinan la intención respecto del e-gobierno, desde una perspectiva conductual. Con ello, se trata de determinar la relevancia de la tendencia identificada por Samuelson y Zeckhauser (1988) que provoca que los individuos no siempre escojan la opción que, supuestamente, les resulta más beneficiosa. Para ello, recurriendo a sesgos cognitivos vinculados al statu quo, se analizaron las relaciones entre el hábito, la inercia, la resistencia y la intención para una muestra de no-usuarios y otra de usuarios de e-gobierno. Partimos de la SQB para la cual el canal que se viene empleando es considerado la alternativa en posesión que condiciona la evaluación de cualquier alternativa (Falk et al., 2007).

Comenzamos con el estudio del hábito puesto que la SQB puede ser vinculada a un hábito generado en la repetición de una acción (Falk et al., 2007; Ng y Kwahk, 2010; Polites y Karahanna, 2012). Respecto al hábito, podemos asumir, como trabajos previos (Ng y Kwahk, 2010; Polites y Karahanna, 2012), que se trata de una fuente inconsciente de inercia, ya que se vinculan ambas variables de forma directa para las dos muestras analizadas. 
La gran diferencia entre las muestras analizadas surge del papel de la inercia, que se relaciona con la intención y resistencia, en ambos casos de forma inversa, para la muestra de no-usuarios, mientras que tales relaciones carecen de significatividad para la muestra de usuarios del e-gobierno. Ello provoca que las relaciones mediadas contempladas en el modelo tan solo resulten significativas para la muestra de no-usuarios. Es decir, para el caso de los no-usuarios del e-gobierno, la resistencia viene determinada por la inercia (Hsieh, 2016; Hsieh y Lin, 2017), al tiempo que para esta muestra se puede aceptar el papel fundamental de la inercia ya que, además de las relaciones directas que presenta, media las relaciones estudiadas (Khedhaouria et al., 2016; Polites y Karahanna, 2012).

Si bien Polites y Karahanna (2012) plantean que una posible motivación de la inercia es la satisfacción, parece que dicha situación no se da para el e-gobierno en España, ya que el superior nivel de satisfacción con el e-gobierno que presentan sus usuarios (AEVAL, 2016), no parece razón suficiente para el desarrollo de la inercia.

En el caso de la muestra de los no-usuarios, la inercia refleja la tendencia al statu-quo, independientemente de las alternativas existentes; describiendo la tendencia a descansar en lo elegido anteriormente, en este caso, mantenerse en el canal que vengan empleando los no-usuarios del e-gobierno (Verplanken y Aarts, 1999), lo cual provoca el estancamiento de las cifras de usuarios del e-gobierno.

Para la muestra de los no-usuarios del e-gobierno, se verifica que los determinantes de la intención son hábito, inercia y resistencia (Khedhaouria et al., 2016; Kim y Gupta, 2012), teniendo presente que tal y como verifica Hsieh (2016), la relación entre inercia e intención de uso es inversa. Asimismo, en ambas muestras la resistencia se vincula con la intención, inversamente con la intención de uso para los no-usuarios y directamente con la intención de continuar con el uso del e-gobierno. En el caso de los usuarios del e-gobierno, no se verifica la relación entre inercia e intención de continuación, es decir, de forma contraria a lo expuesto por Olsen et al. (2013), conforme se desarrolla la relación, la continuación no viene determinada por la inercia.

Por lo expuesto, en línea con Anderson (2003), entre las causas que llevan a los ciudadanos a no emplear el e-gobierno, cambiando su canal de acceso a los servicios públicos, se encuentran la SQB, la inercia a la inacción y la resistencia del consumidor a las innovaciones. De este modo, la SQB se manifiesta, finalmente, como una inercia que refleja la carencia de deseo por abandonar la alternativa en uso independientemente de las alternativas presentes o de aquellas que pudieran aparecer.

\section{CONCLUSIONES, LIMITACIONES Y FUTURAS LÍNEAS DE INVESTIGACIÓN}

El presente trabajo se ha desarrollado con el objetivo de establecer la aplicabilidad de enfoque conductual en el uso del e-gobierno. Para ello, y con el objetivo de alcanzar una mayor profundidad en los resultados, se ha comparado los resultados de dos muestras, una de no-usuarios y otra de usuarios del e-gobierno.

En la muestra de los no-usuarios del e-gobierno, se verifica el funcionamiento de los sesgos tal y como establece la literatura con relaciones entre hábito, inercia y resistencia y de éstos con la intención de uso del e-gobierno. Asimismo, resultan significativas las relaciones mediadas incorporadas en el modelo.

Para el uso de la muestra de usuarios del e-gobierno, la intención de continuación en su uso viene determinada por hábito y resistencia, pero carecen de significación aquellas relaciones en las que participa el hábito.

Es por ello que, respondiendo al objetivo del trabajo, se constata la presencia de la SQB en el uso del e-gobierno. Una tendencia que mientras en el caso de los no-usuarios del e-gobierno lleva a dilatar su adopción, en los usuarios provoca una permanencia en el uso del e-gobierno como canal de acceso a los servicios públicos.

Pese a que se ha venido señalando que los procesos cognitivos son los determinantes del comportamiento, en la práctica muchos comportamientos cotidianos, como puede ser la elección del canal a través del cual interactuar con la administración, se producen con muy poca deliberación cognitiva. Para que se produzca el cambio en el comportamiento de los ciudadanos debemos actuar sobre los hábitos (Moraes et al., 2012).

Como se puede observar en los resultados, el hábito juega un papel fundamental sobre la intención vinculada al uso del e-gobierno. Es por ello que comenzaremos las implicaciones por las diferentes intervenciones existentes. Así, se podría recurrir a campañas descendentes, para aquellos casos en los que determinados grupos de ciudadanos se encuentren en riesgo de caer en la brecha digital. La Estrategia de Territorios Inteligentes del gobierno de España presenta como tercer ámbito de actuación los denominados servicios públicos 4.0, apoyados en soluciones tecnológicas que filtren la oferta según las necesidades del 
ciudadano. Esto es, se tiene presente la posibilidad de desarrollar diferentes estrategias en función del tipo de ciudadano que se dirija. Cabe recordar que la aplicación de la economía conductual al sector público considera adecuada la aplicación de técnicas de segmentación en las que las actuaciones se dirijan a un grupo de la población (OECD, 2017).

Para los no-usuarios del e-gobierno resultarían de aplicación campañas que comuniquen los beneficios vinculados al mismo. Cabe recordar que la resistencia de los no-usuarios al uso del e-gobierno puede venir motivada por los costes de cambio (Bhattacherjee y Park, 2014). Por ello, las citadas campañas de comunicación podrían trabajar en esta línea, ya que su uso encuentra apoyo en la aplicación del enfoque coonductual en el sector público (OECD, 2017).

Dentro de las campañas ascendentes, cabe recordar dos herramientas vinculadas a la aplicación de los principios coonductuales a la administración pública (Grune-Yanoff y Hwetwig, 2016; OECD, 2017): los estímulos a la decisión, también denominados nudges (codazos), interacciones que dirigen a las personas en una dirección particular al tiempo que se preserva su libertad de elección; y, las intervenciones denominadas «impulsores», cuyo objetivo es reforzar la capacidad de los ciudadanos para tomar sus propias decisiones.

Los posibles cambios normativos entrarían dentro de las intervenciones ascendentes, que se valen de cambios del entorno, aprovechando que la mayor parte del comportamiento humano puede ser explicado por el entorno de la tarea a realizar. En esta línea se trabaja en la Ley 15/2014, de 16 de septiembre que contempla la implantación de procedimientos electrónicos para que se reduzcan las trabas burocráticas e impulsen una administración más cercana a los ciudadanos y empresas. Busca lograr una administración totalmente electrónica e interconectada y reducir las cargas administrativas. Es decir, busca conseguir la simplificación de los procedimientos para ciudadanos y empresas, reduciendo las trabas burocráticas e impulsando el e-gobierno (Munuera, 2016).

Asimismo, se podría actuar mediante intervenciones descendientes con cambios en el entorno, en el que se aprovecha que los ciudadanos se enfrentan a nuevas situaciones. En este caso, se debería actuar sobre procesos transversales a los diferentes grupos de ciudadanos.

Para cualquiera de las actuaciones citadas, debemos tener presente que el cambio del hábito será más complejo en el caso de que el ciudadano haya repetido el comportamiento en numerosas ocasiones. Por ello, se sugiere actuar, en la medida de lo posible en el momento en el cual se comienza a desarrollar un comportamiento. En esta línea, la Ley 39/2015 señala que la tramitación electrónica debe constituir la actuación habitual de las administraciones, a través del establecimiento de derechos y obligaciones concretos. En el caso de las personas físicas, se «mantiene el derecho a elegir la forma de relación con la administración, ya sea por la vía telemática o por los medios convencionales», esto es, derecho a elegir el canal. Para otros colectivos, expuestos en el artículo 14 de la citada ley, se establece la obligatoriedad de que se relacionen con la Administración por la vía electrónica.

Con el objetivo de generar habitualidad, se presentan opciones como Cl@ve, un sistema orientado a simplificar el acceso electrónico de los ciudadanos a los servicios públicos, permitiendo que puedan identificarse ante la administración mediante claves concertadas. Permitirá la firma electrónica reconocida con certificado en la nube e integración con el DNI3.0, facilitando a los ciudadanos el acceso al e-gobierno (Munuera, 2016).

A ella se le unen otros elementos que pueden favorecer el desarrollo de hábito e inercia para la adopción del e-gobierno como pueden ser el punto general de entrada de facturas de la administración del estado, que ofrece a los proveedores un punto común para todas las administraciones públicas para la presentación de facturas electrónicas; la interconexión de registros y la eliminación del soporte papel, que elimina el tránsito de papel entre oficinas, aumentando la eficiencia y eliminando los costes de manipulación y remisión del papel; o la carpeta ciudadana, que permite consultar de manera personalizada en un único punto todos los expedientes de un ciudadano, así como sus notificaciones administrativas, los datos intermediados por la administración, los asientos registrales y el acceso a servicios horizontales como apoderamientos o documentos (Vidal y Delgado, 2017: 52).

Si se quiere provocar que los ciudadanos accedan a los servicios públicos a través del e-gobierno, se podría trabajar con elementos relativos a la satisfacción, al haber sido identificada como una posible fuente de inercia (Polites y Karahanna, 2012). Se propone profundizar en un trabajo ya en marcha, ya que, en los últimos años, las dos dimensiones de la satisfacción que más mejoran son la incorporación de nuevas tecnologías (SMS, citas telefónicas automáticas, uso de internet), y la posibilidad de acceso a los servicios públicos a través de internet, pese a lo cual siguen siendo las dimensiones menos relacionadas con el nivel de 
satisfacción general con los servicios públicos. Es decir, los ciudadanos las valoran, pero no llegan a percibir a las nuevas tecnologías como elementos nucleares dentro de la prestación del servicio. A este respecto, serían de aplicación las campañas de comunicación antes referidas.

\section{REFERENCIAS BIBLIOGRÁFICAS}

AGENCIA DE EVALUACIÓN Y CALIDAD (AEVAL). (2016). Estabilidad y mejora en los servicios públicos. Ministerio de Hacienda y Administración Pública.

ANDERSON, C. J. (2003), "The Psychology of Doing Nothing: Forms of Decision Avoidance. Results from Reason and Emotion", Psychological Bulletin, 129 (1): 139-167. DOI: https/doi.org/10.1037//0033-2909.129.1.139.

ARIELY, D. (2008). Predictably Irrational: The Hidden Forces That Shapes our Decisions. NY: Harper Collins.

BHATTACHERJEE, A. (2001). "Understanding Information Systems Continuance. An Expectation-Confirmation Model". MIS Quarterly, 25 (3), 351-370. https://doi.org/10.2307/3250921.

BHATTACHERJEE, A. y Hikmet, N. (2007), "Physicians' resistance toward healthcare information technology: A theoretical model and empirical test", European Journal of Information Systems, 16: 725-737. https/doi. org/10.1057/palgrave.ejis.3000717.

BHATTACHERJEE, A. y LIN, C.-P. (2015), "A unified model of IT continuance: three complementary perspectives and crossover effects", European Journal of Information Systems, 23: 364-373. https/dx.doi.org/10.1016/j. emj.2011.11.003.

BHATTACHERJEE, A. y PARK, A. C. (2014), "Why end-users move to the cloud: a migration-theoretic analysis", European Journal of Information Systems, 23: 357-372. https://doi.org/10.1057/ejis.2013.1.

CASTELNOVO, W. y SORRENTINO, M. (2017), "The digital government imperative: a context-aware perspective", Public Management Review, article in press. https://doi.org/10.1080/14719037.2017.1305693.

CHOUEIKI, A. (2016), "Behavioral Insights for Better Implementation in Government", Public Administration Review, 76 (4): 540-541. https://doi.org/10.1111/puar.12594.

DATTA, S. y MULLAINATHAN, S. (2014), "Behavioral design: a new approach to development policy", Review of Income and Wealth, 60 (1): 7-35. https://doi.org/10.1111/roiw.12093.

DWIVEDI, Y. K., RANA, R. P., JANSSEN, M., LAL, B. WILLIAMS, M. D. y CLEMENT, M. (2017), "An empirical validation of a unified model of electronic government adoption (UMEGA)", Government Information Quarterly, 34: 211-230. http://dx.doi.org/10.1016/j.giq.2017.03.001.

EBBERS, W. E. y VAN DIJK, J. A. G. M. (2007), "Resistance and support to electronic government, building a model of innovation", Government Information Quarterly, 24: 554-575. https://doi.org/10.1016/j.giq.2006.09.008.

FALK, T., SCHEPERS, J., HAMMERSCHMIDT, M. y BAUER, H. H. (2007), "Identifying Cross-Channel Dyssinergies for Multichannel Service Providers", Journal of Service Research, 10 (2): 143-160. https://doi. org/10.1177/1094670507306683.

GRIMMELIKHUIJSEN, S., JILKE, S., OLSEN, A. L. y TUMMERS, L. (2016), "Behavioral Public Administration: Combining Insights from Public Administration and Psychology", Public Administration Review, 77 (1): 45-56. https://doi.org/10.1111/puar.12609.

GRUNE-YANOFF, T. y HERTWIG, R. (2016), "Nudge Versus Boost: How Coherent are Policy and Theory?", Minds and Machines, 26 (1-2): 149-183. https://doi.org/10.1007/s11023-015-9367-9.

HAIR, J. F., RINGLE, C. M. y SARSTEDT, M. (2011). PLS-SEM: Indeed a Silver Bullet. Journal of Marketing Theory and Practice, 19 (2), 139-151. https://doi.org/10.2753/mtp1069-6679190202.

HSIEH, P. J. y LIN, W. S. (2017), "Explaining resistance to system usage in the PharmaCloud: A view of the dual-factor model. Information \& Management". Article in press. $h t t p: / / d x . d o i . o r g / 10.1016 / j . i m .2017 .03 .008$.

HSIEH, P.-J. (2016), "An empirical investigation of patients' acceptance and resistance toward the health cloud: the dual factor perspective", Computers in Human Behavior, 63: 959-969. https://doi.org/10.1016/j.chb.2016.06.029.

JILKE, S., VAN DE WALLE, S. \& KIM, S. (2016). Generating Usable Knowledge Through an Experimental Approach to the Public Administration. Public Administration Review, 76 (1), 69-72. https://doi.org/10.1111/puar.12488.

KAHNEMAN, D., KNETSCH, J. L. y THALER, R. H. (1991), "Anomalies: The Endowment Effect, Loss Aversion, and Status Quo Bias", Journal of Economic Perspectives, 5 (1): 695-707. https://doi.org/10.1257/jep.5.1.193.

KHEDHAOURIA, A., THURIK, R., GURAU, C. y van HECK, E. (2016), "Customers' Continuance Intention Regarding Mobile Service Providers: A Status Quo Bias Perspective", Journal of Global Information Management, 24 (4): 1-21. https://doi.org/10.4018/jgim.2016100101.

KIM, H.-W. y GUPTA, S. (2012), "Investigating Customer Resistance to Change in Transaction Relationship with an Internet Vendor", Psychology \& Marketing, 29 (4): 257-269. https://doi.org/10.1002/mar.20519.

KIM, H.-W. y KANKANHALLI, A. (2009), "Investigating User Resistance to Information Systems Implementation: A Status Quo Bias Perspective", MIS Quarterly, 33 (3): 567-582. https://doi.org/10.2307/249384.

LEE, K. y JOSHI, K. (2016), "Examining the use of status quo bias perspective in IS research: need for reconceptualizing and incorporating biases", Information Systems Journal. https://doi.org/10.1111/isj.12118. 
LI, Z. y CHENG, Y. (2014), "From free to fee: exploring the antecedents of consumer intention to switch to paid online content", Journal of Electronic Commerce Research, 15 (4): 281-299.

LIMAYEN, M., HIRTZ, S. G. y CHEUNG, C. M. K. (2007), "How habit limits the predictive power of intention: the case of information systems continuance", MIS Quarterly, 31 (4): 705-737. https://doi.org/10.2307/3250921.

LUNA-REYES, L. F., HERNÁNDEZ GARCÍA, J. M. y GIL-GARCÍA, J. R. (2009), "Hacia un modelo de los determinantes de éxito de los portales de gobierno estatal en México", Gestión y política pública, 18 (2): $307-340$. Disponible en web: http://www.scielo.org.mx/scielo.php?script=sci_arttext\&pid=S1405-10792009000200003.

MAIO, G. R., VERPLANKEN, B., MANSTEAD, A. S., STROEBE, W., ABRAHAM, C., SHEERAN, P. y CONNER, M. (2007), "Social psychological factors in lifestyle change and their relevance to policy", Social Issues and Policy Review, 1 (1): 99-137. https://doi.org/10.1111/j.1751-2409.2007.00005.x.

MEDINA, C., RUFIN, R. y REY, M. (2013), "La Adopción Del E-Gobierno En Entornos Voluntarios", Investigaciones Europeas de Dirección y Economía de la Empresa, 19 (1), 42-52. http://dx.doi.org/10.1016/j. iedee.2012.10.001.

MEDINA, C. (2017). Estrategia omnicanal en la distribución de servicios públicos. Madrid: Instituto Nacional de Administración Pública.

MEDINA-MOLINA, C., REY-MORENO, M. y PÉREZ-GONZÁLEZ, B. (2017), "Estrategia omnicanal para no usuarios del e-gobierno", GAPP, 18 (noviembre): 94-109. https://doi.org/10.24965/gapp.v0i18.10463.

MORAES, C., CARRIGAN, M. y SZMIGIN, I. (2012), "The coherence of inconsistencies: Attitude-behaviour gaps and new consumption communities", Journal of Marketing Management, 28 (1-2): 103-128. https://doi.org/10.1080/026 7257x.2011.615482.

MOYNIHAN, D. P. y LAVERTU, S. (2012), "Cognitive biases in governing: Technology preferences in election administration", Public Administration Review, 72 (1): 68-77. https://doi.org/10.1111/j.1540-6210.2011.02478.x.

MUNUERA, M. P. (2016), "Agenda digital: e-Servicios sociales", Gestión y Análisis de Políticas Públicas, 16 (juliodiciembre): 27-40. https://doi.org/10.24965/gapp.v0i16.10362.

NG, E. H. y KWAHK, K. Y. (2010), "Examining the determinants of Mobile Internet service continuance: A customer relationship development perspective", International Journal of Mobile Communications, 8 (2): 210-229. https://doi. org/10.1504/ijmc.2010.031448.

NYGREN, K. G., AXELSSON, K. y MELIN, U. (2014), "Multi-Channel Service Management in Public Sector - Three Interpretative Frames Illustrating E-government and Work Practice in a Swedish State Agency", Electronic Journal of e-Government, 12 (1): 115-128. Disponible en: http://www.ejeg.com/volume12/issue1.

OECD (2015), Behavioural insights and new approaches to policy design. The views from the field. Summary of an

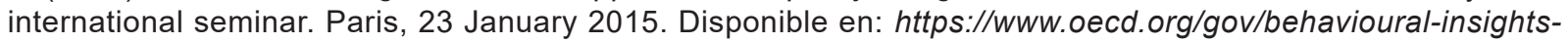
summary-report-2015.pdf.

OECD (2017), Behavioural Insights and Public Policy: Lessons from Around the World, OECD Publishing, Paris. http:// dx.doi.org/10.1787/9789264270480-en.

OLSEN, S. O., TUDORAN, A. A., BRUNSO, K. y VERBEKE, W. (2013), "Extending the prevalent consumer loyalty modelling: the role of habit strength", European Journal of Marketing, 47 (1-2): 303-323. https://doi. org/10.1108/03090561311285565.

ONTSI (2017). Indicadores destacados de la Sociedad de la Información.

ORTIZ DE GUINEA, A. y MARKUS, M. L. (2009), "Why break the habit of a lifetime? Rethinking the roles of intention, habit, and emotion in continuing information technology use", MIS Quarterly, 33 (3): 433-444. Disponible en: http:// www. misq.org/contents-33-3/.

PATSIOTIS, A., HUGHES, T. y WEBBER, D. J. (2013), "An examination of consumers' resistance to computer based technologies", Journal of Service Marketing, 27 (4): 294-312. https://doi.org/10.1108/08876041311330771.

POLITES, G. L. y KARAHANNA, E. (2012), "Shackled to the Status Quo: the inhibiting effects of incumbent system habit, switching costs, and inertia on new system acceptance", MIS Quarterly, 36 (1): 21-42. Disponible en: http:// www.misq.org/contents-36-1/.

PONT, J. (2016), "Modelos innovadores de administración y gestión pública: Hacia la emergencia de nuevos paradigmas", Gestión y Análisis de Políticas Públicas, 16 (julio-diciembre). https://doi.org/10.24965/gapp. v0i16.10364.

ROLDÁN, J. L. y SÁNCHEZ-FRANCO, M. J. (2012). "Variance-based Structural Equation Modeling: Guidelines for Using Partial Least Squares in Information Systems Research". En MORA, M. et al. (Eds.) Research Methodologies in Engineering of Software Systems and Information Systems: Philosophies, Methods and Innovations. Hershey, PA: IGI Global. (Chapter II.3).

SAMUELSON, W. y ZECKHAUSER, R. (1988), "Status Quo Bias in Decision Making", Journal of Risk and Uncertainty, 1: 7-59. https://doi.org/10.1007/bf00055564.

SCHÄFER, M., JAEGER-ERBEN, M. y BAMBERG, S. (2012), "Live Events as Windows of Opportunity for Changing Towards Sustainable Consumption Patterns?”, Journal of Consumer Policy, 35: 65-84. https://doi.org/10.1007/ s10603-011-9181-6.

SIMON, H. A. (1955). "A Behavioral Model of Rational Choice”. The Quaterly Journal of Economics, 69: 99-118. https:// doi.org/10.2307/1884852. 
TAYLOR, S. y TODD, P. (1995), "Understanding information technology usage: A test of competing models", Information Systems Research, 6 (2): 144-176. https://doi.org/10.1287/isre.6.2.144.

TRIANDIS, H. C. (1980), Values, attitudes, and interpersonal behaviour, 27. Lincoln: Nebraska: University of Nebraska Press.

VAN VEENSTRA, A. y JANSSEN, M. (2010), "Migration Strategies for Multi-Channel Service Provisioning in Public Agencies", Electronic Journal of e-Government, 8 (2): 215-226. Disponible en: http://www.ejeg.com/volume8/ issue2.

VENKATESH, V., THONG, J. Y., CHAN, F. K., HU, P. J. H. y BROWN, S. A. (2011), "Extending the two-stage information systems continuance model: Incorporating UTAUT predictors and the role of context", Information Systems Journal, 21 (6): 527-555. https://doi.org/10.1111/j.1365-2575.2011.00373.x.

VERPLANKEN, B. y AARTS, H. (1999), "Habit, attitude, and planned behavior: Is habit an empty construct or an interesting case of goal-directed automaticity?", en STROEBE, W. y HEWSTONE, M. (Eds.), European Review of social psychology, 10 (1): 101-134.

VERPLANKEN, B. y WOOD, W. (2006), "Interventions to Break and Create Consumer Habit", Journal of Public Policy \& Marketing, 25 (1): 90-103. https://doi.org/10.1509/jppm.25.1.90.

VIDAL, C. y DELGADO, D. (2017), "La reforma de las Administraciones Públicas y el Estado Social en la crisis económica (2012-2015)", Gestión y Análisis de Políticas Públicas, 17 (mayo): 40-62. https://doi.org/10.24965/ gapp.v0i17.10400.

WANG, Q., SONG, P. y YANG, X. (2013), "Understanding the substitution effect between online and traditional channels: evidence from product attributes perspectives", Electronic Markets, 23: 227-239. https://doi.org/10.1007/ s12525-012-0114-2.

WANG, R. J. H., MALTHOUSE, E. C. y KRISHNAMURTHI, L. (2015), "On the Go: How Mobile Shopping Affects Customer Purchase Behavior", Journal of Retailing, 91 (2): 217-234. https://doi.org/10.1016/j.jretai.2015.01.002.

WOOD, W. y NEAL, D. T. (2006), "The habitual consumer", Journal of Consumer Psychology, 19: 579-592. https://doi. org/10.1016/j.jcps.2009.08.003. 